\title{
APROXIMACIÓN AL ESTUDIO \\ DE LAS DIFERENCIAS INDIVIDUALES \\ Y AL DIAGNÓSTICO DE PATOLOGÍA DUAL \\ DESDE LA PERSPECTIVA PSICOLÓGICA \\ EN NIÑOS Y ADOLESCENTES \\ CON SÍNDROME DE DOWN
}

\section{An Approach to the Study of Individual Differences and the Dual Diagnosis from Psychological Perspective in Children and Adolescents Down Syndrome}

Gracia Delgado-Pardo

Universidad de Sevilla. Facultad de Psicología. Departamento Personalidad, Evaluación y Tratamiento Psicológicos

gdelgado1@us.es

Recepción: 4 de agosto de 2020

Aceptación: 26 de enero de 2021

RESUMEN: La cada vez mayor inclusión social, la normalización de contextos y los avances de la investigación han contribuido sobremanera a la mejora de las expectativas de vida y a la participación integral en todos los espacios de la sociedad por parte de las personas con síndrome de Down (SD). Sin embargo, durante décadas la identificación de síntomas psicológicos o psiquiátricos ha resultado una tarea complicada al atribuirse estos como correlatos propios de la discapacidad intelectual. Con este interés, se han revisado las publicaciones científicas desde 1930 hasta 2020, con el propósito de: 1) examinar las investigaciones que, desde la perspectiva de las diferencias individuales, analicen la evolución y los cambios producidos en la concepción del SD y, desde este enfoque, 2) investigar la prevalencia y manifestación clínica de patología dual junto al SD, en población infantil y adolescente. Entre las conclusiones se destaca la necesidad de cambio, por parte de profesionales y familias, para la asunción de la existencia y aceptación del 
diagnóstico dual que posibilite diagnósticos precoces e intervención psicológica ajustada a esta demanda.

Palabras Clave: niños y adolescentes; síndrome de Down; patología dual; diferencias individuales; trastorno psicológico.

ABSTRACT: Ever-increasing social inclusion, normalisation and progress made in research have significantly contributed to improving the life expectancy of those with Down syndrome (DS) and have seen them take on a more active and fuller role within all areas of society. For decades, the all-encompassing view of the syndrome meant that issues relating to mental health and psychological well-being were often overlooked, hidden under the guise of intellectual disability. It is within this context that scientific publications on the topic since 1930 through to 2020 have been reviewed in order to: 1) examine the research available which, based on individual differences, analyses the progress made and changes in terms of how DS is seen, and based on this perspective, 2) research the prevalence and clinical symptoms of dual diagnosis involving DS in children and teenagers. Based on the conclusions of this review, the need for professionals and families to acknowledge the existence of and accept dual diagnosis is apparent, something which would allow for early diagnosis and psychological intervention appropriate for the individual's needs.

KEYWORDS: children and adolescents; Down Syndrome; dual pathology; individual differences; psychological disorder/psychiatric disorder.

\section{Introducción}

4

L SÍNDROME DE Down (SD) continúa siendo la cromosomopatía, de causa conocida, de mayor incidencia a nivel mundial y el motivo más frecuente de discapacidad intelectual congénita y, con ello, el más ampliamente investigado (HueteGarcía, 2016; Startin et al., 2020).

Tradicionalmente, el abordaje y el estudio de los aspectos descriptivos del síndrome se han realizado bajo la concepción de "grupo homogéneo" (Gibbs y Thorpe, 1983; Rodgers, 1987; Wishart y Johnston, 1990). Se trata de una concepción preconcebida por la que se les presupone unos rasgos neonatales característicos determinantes de una apariencia física similar, el padecimiento de cierto tipo de patologías y otros problemas médicos derivados del síndrome (Flórez y Ruiz, 2004). Igualmente, esta visión globalizadora se refiere además a aspectos descriptivos que tienen que ver con formas de ser y actuar, rasgos de carácter y de personalidad, así como respuestas sociales que serían semejantes o muy parecidas entre ellos y que se relacionarían también con el origen genético del síndrome, estando influenciadas por el grado de discapacidad intelectual (Campbell et al., 2003).

Con el tiempo, esta visión común globalizadora sobre el SD ha ido transformándose, cediendo espacio a otras perspectivas de estudio alejadas de la idea del grupo homogéneo y que coincide, también, con la ocurrencia de otro tipo de cambios y 
avances. Por ejemplo, se destaca la evolución sobre la conceptualización del contexto social. En los años 60 y 70 del pasado siglo XX, muchas personas con discapacidad intelectual vivían recluidas en instituciones y alejadas de su núcleo familiar bajo la creencia, común y normalizada, de la existencia de una asociación directa entre el grado de discapacidad intelectual y la presencia de enfermedad mental, hecho común también entre las personas con SD (Paterson, 1999). Sin embargo, a raíz del proceso de desinstitucionalización y junto al cambio de conceptualización sobre la discapacidad intelectual, se consigue transformar esta idea de solapamiento entre ambas condiciones, enfermedad mental y discapacidad intelectual, lográndose que estas personas se incorporen a vivir en sociedad, con los mismos derechos que el resto de la población y con total acceso a los servicios comunitarios (Glasson et al., 2014).

De igual modo, se ha producido un aumento significativo de la esperanza de vida de las personas con SD, gracias a los avances médicos y a la mejora de los tratamientos de comorbilidades que han repercutido en una reducción importante de la mortalidad en los primeros años de vida (Weijerman et al., 2008; Uppal et al., 2015). Y junto a ello, el desarrollo de la investigación sobre los procesos de envejecimiento y las enfermedades degenerativas, tales como la demencia y el alzhéimer, que han propiciado también una mejora, en este sentido, de la calidad de vida de las personas con SD (Karmiloff-Smith et al., 2016; Coppus, 2017).

De manera que, en un contexto psicosocial normalizado e inclusivo, y a raíz de los cambios observados, surge la necesidad de prestar atención a la salud mental de esta población, en general, y a los trastornos de origen psicológico, en especial, ya que, con anterioridad, solían pasar desapercibidos por el solapamiento y/o la sobreprotección e incluso por el desconocimiento, por parte de los padres, pero, sobre todo, de los propios profesionales (Paterson, 1999; Dykens, 2007; Garvía, 2014; Hartaway, 2016). En la actualidad, se ha demostrado que la afectación de la salud mental aparece en mayor proporción en la población con discapacidad intelectual que en la población general, siendo su detección y diagnóstico más complicados a causa de las diferencias en la expresión y manifestación de síntomas, así como por la falta de recursos específicos disponibles para su abordaje (Ortiz, 2012; Garvía, 2017).

Por todo ello, el objetivo principal de esta investigación se define en dos focos principales de atención: a) explorar las investigaciones que, desde la perspectiva de las diferencias individuales, analizan la evolución y los cambios producidos en las personas con SD y, bajo este enfoque, b) examinar la prevalencia y la manifestación clínica de la patología dual, esto es, la coexistencia de síntomas y/o trastorno psicológico o enfermedad psiquiátrica junto al SD en población infantil y adolescente.

Para alcanzar ambos objetivos, se ha realizado una búsqueda bibliográfica a través de la plataforma ISI Web of Knowledge incluyendo el periodo comprendido entre 1930 y la actualidad, abril de 2020. Al tratarse de menores (población infantil y adolescente), se han empleado, entre otros, los siguientes descriptores: síndrome de Down, niños, adolescentes, fenotipo conductual, genético, trastorno dual, comorbilidad, diferencias individuales, trastorno de espectro autista (TEA), autismo, trastorno por déficit de atención e hiperactividad (TDAH), déficit de atención, trastorno de la conducta, impulsividad, ansiedad y trastornos afectivos, catatonia, trastorno desinte- 
APROXIMACIÓN AL ESTUDIO DE LAS DIFERENCIAS INDIVIDUALES Y AL DIAGNÓSTICO DE PATOLOGÍA DUAL DESDE LA PERSPECTIVA PSICOLÓGICA EN NIÑOS Y ADOLESCENTES CON SÍNDROME DE DOWN G. DELGADO-PARDO

grativo. En la combinación de diferentes variantes e introduciendo los términos tanto en español como en inglés.

El análisis de la información obtenida tras la revisión se ha agrupado en dos apartados que responden a los objetivos inicialmente planteados en este trabajo.

\section{Diferencias individuales en los niños y adolescentes con síndrome de Down}

En un primer acercamiento, las publicaciones sobre características descriptivas de las personas con SD se aproximan, predominantemente, a la corriente de estudio basada en el enfoque del estereotipo (Pueschel y Myers, 1994). Es cierto que el origen sindrómico da lugar a la presencia de características comunes en cuanto a salud física y rasgos descriptivos similares en las personas con $\mathrm{SD}$, de manera que con frecuencia se les describe en cuanto a su carácter como personas cariñosas, amistosas y afectuosas, con rasgos de personalidad positivos que prevalecen sobre los negativos (Fidler et al., 2005; Fidler, 2006; Rondal y Ling, 2006).

Gibbs y Thorpe, en 1983, confirmaban esta idea; al comparar entre sí niños con SD (sin institucionalizar) con un grupo control, concluían afirmando que el estereotipo clásico estaba presente, independientemente del género y de la raza del menor, desprendiéndose una base objetiva sobre este concepto. Rodgers (1987) investigó la concordancia materna con respecto al estereotipo en los menores con SD, de manera que al pedirles a madres de niños entre 3 y 9 años que describieran a los niños con SD lo hacían en base a atributos de personalidad estereotipados, quizá, influenciadas por la familiaridad del síndrome. Si se trataba de las madres de los menores con SD aumentaba el número de atributos de rasgos de personalidad. Años después, Wishart y Johnston (1990) construyeron un cuestionario basado en adjetivos de personalidad asociados a niños con SD que fue administrado a un grupo de adultos. Entre sus hallazgos obtuvieron que los adultos con un contacto más frecuente con esta población eran significativamente menos propensos a clasificar estereotípicamente a los niños con SD, frente a aquellos que referían poco contacto. Entre los participantes se encontraban las madres de los niños, estudiantes de Medicina, Psicología y maestros de primaria (con diferentes niveles de relación con los menores). Sin embargo, y a pesar de los resultados, la investigación concluía aceptando la noción del estereotipo de personalidad, se les infería una idea de identidad común en contraste con los niños de desarrollo típico a los que se les atribuían identidades individuales distintas. Zickler et al. (1998) se refirieron a las características únicas de los niños con SD en primera infancia (1-4 meses), para que fueran tenidas en cuenta por las enfermeras que les atendían destacando que, aunque constituyeran un grupo homogéneo, no eran tan diferentes de sus iguales de desarrollo normativo. Collacott et al. (1998) confirmaban la existencia de un fenotipo de comportamiento característico en adultos con SD bajo la demanda de mayor investigación al respecto.

En nuestro ámbito, también ha sido común la aceptación del concepto de grupo homogéneo. Los autores confirmaban la apreciación de aspectos semejantes en la forma de ser y actuar en las personas con SD, que permitían señalar algunas característi- 
APROXIMACIÓN AL ESTUDIO DE LAS DIFERENCIAS INDIVIDUALES Y AL DIAGNÓSTICO DE PATOLOGÍA DUAL DESDE LA PERSPECTIVA PSICOLÓGICA EN NIÑOS Y ADOLESCENTES CON SÍNDROME DE DOWN G. DELGADO-PARDO

cas propias en distintas funciones psicológicas (Arranz, 2002; Troncoso et al., 1999) y por las que estas personas obtenían, igualmente, un buen grado de adaptación social al ofrecer una imagen social más favorable que en el caso de otras discapacidades (Flórez y Ruiz, 2004).

Sin embargo, comienza a observarse un cambio puesto que, independientemente de la apariencia similar de las personas con SD por el hecho de compartir la trisomía en el par 21, se da en ellos una gran heterogeneidad determinada por la herencia, el ambiente o contexto de desarrollo y el porcentaje de discapacidad intelectual de cada uno de ellos (Basile, 2008; Korenromp et al., 2007). De manera que presuponer unos rasgos propios identificativos de las personas con SD implica dos riesgos, la generalización y la creación de expectativas, que en definitiva limitan sus opciones ofreciendo una visión globalizadora y perpetuadora de actitudes y creencias incompatibles con la posibilidad de un mayor desarrollo e inclusión social (Santiago y Marrero, 2003).

En la misma línea, el progresivo avance de la investigación sobre el fenotipo conductual en los trastornos de origen genético se ha orientado hacia la perspectiva de las diferencias individuales, por ejemplo, Dykens (1995) definía en términos probabilísticos el fenotipo conductual, al referir que, aunque fuera más probable que las personas que compartían un determinado síndrome mostraran comportamientos comunes característicos en comparación con otras personas con diferentes discapacidades del desarrollo, sin embargo, y en función de la variabilidad, no todos los sujetos con una alteración genética común tendrían que poseer las mismas características típicas de ese trastorno, ni en el mismo grado, sino que debería de tenerse en cuenta la interrelación que puede darse entre genes, ambiente y experiencia.

En este sentido, Cebula et al., en 2010, se referían a la escasez de estudios sobre el desarrollo de los procesos sociales en la discapacidad intelectual y, en concreto en el $\mathrm{SD}$, relacionándolo con la visión estereotipada sobre la personalidad sociable de estas personas que provocaba que se entendiera el desarrollo de lo social, en ellos, como un proceso invariable. Mientras que, por el contrario, en relación con la población general el estudio de los procesos sociales se consideraba como un importante reforzador del desarrollo cognitivo de los niños. En definitiva, comienza a ponerse de relieve el cambio que se estaba produciendo en cuanto al desarrollo del estudio del fenotipo conductual y el consecuente desarrollo del estudio de la cognición social en la discapacidad intelectual y en los síndromes genéticos, atendiendo al perfil preciso de desarrollo de estos sujetos (Fidler, 2005; Fidler et al., 2006).

Los autores, bajo esta orientación, comienzan a plantearse cómo también las personas con SD muestran marcadas diferencias individuales en habilidades cognitivas debidas a aspectos genéticos, pero también a los ambientales (Määttä et al., 2006). En relación con población infantil, McCarthy (2008) señaló que era posible que existieran ciertos marcadores de riesgo en la infancia, en concreto, características individuales y factores ambientales que pudieran contribuir a predecir el desarrollo de trastornos de conducta grave en adultos jóvenes con SD. Grieco et al. (2015) publicaron una revisión donde se actualizaban los datos sobre fenotipos cognitivos y conductuales, asociados al SD y observados en todos los periodos evolutivos de la persona. Ellos partían de la concepción de la existencia de perfiles neurocognitivos y neuroconduc- 
tuales únicos que emergerían en periodos específicos del desarrollo particular de cada individuo con SD, diferentes a los de otros tipos de discapacidad intelectual similar, y que originarían un perfil fenotípico distinto. Por su parte, Coppus (2017), para demostrar los progresos sobrevenidos por los avances en los tratamientos y la mejora de la calidad de vida, realizó un estudio longitudinal de cohorte prospectivo con el que poder comparar dos generaciones de manera transversal. Los resultados mostraron diferencias entre ambos grupos relacionados con las mejoras en los cuidados de la salud y los cambios producidos en el ambiente, siendo todos estos cambios más beneficiosos para los adultos de generaciones venideras. Karmiloff-Smith et al. (2016) enfatizaron en esta misma idea, sobre la necesidad de estudiar la trisomía 21 en profundidad y a todos los niveles (genético, celular, neuronal, cognitivo, conductual y ambiental), entendiendo que es el mejor enfoque para poder comprender el genotipo y su relación con el fenotipo; y, a partir de ahí, poder explorar factores de riesgo para el desarrollo de otras enfermedades cada vez más comunes en los adultos mayores con $\mathrm{SD}$, como es el alzhéimer.

En España, ocurre también este cambio de concepción, sobre la aceptación de la variabilidad de los rasgos fenotípicos entre las personas con SD. Flórez y Dierssen (2006) se referían a la posible variabilidad física y cognitiva que podría llegar a ser considerable en estas personas, contando con las características propias del síndrome y los grados distintos de discapacidad intelectual. Junto a ello, los diferentes ambientes familiares, educativos y sociales también influirían en el desarrollo físico y psicológico de esta población. Flórez se refiere a diferentes motivos para la perpetuación de la idea de grupo homogéneo, pero sostiene que este es el momento de aceptar definitivamente el cambio. La justificación del mismo la expone en base a la investigación disponible sobre las diferencias individuales presentes en las personas con SD en cuanto a su estructura cerebral, genética y desarrollo de la cognición que, insiste, han de ser tenidas en cuenta (Flórez et al., 2015; Flórez, 2019).

\section{Patología dual en el niño y adolescente con síndrome de Down}

El término patología o diagnóstico dual, utilizado para referirse a la confluencia de trastorno psicológico o enfermedad mental junto a discapacidad intelectual, es un concepto que cuenta hasta el momento con una amplia trayectoria, al menos, en el ámbito de la investigación (Hernández et al., 2011). En este sentido, las publicaciones coinciden en cómo esta circunstancia de afectación a la salud mental es, incluso, más común de lo esperado en personas con discapacidad intelectual, situándose las cifras de prevalencia por encima de lo observado en la población general (Vicari et al., 2013; Warner et al., 2017). Según datos de nuestro entorno proporcionados por la ENSE 2017, la prevalencia estimada para población normativa de trastorno mental infantil (ansiedad y depresión) (0-14 años) y autismo (3-14 años) es del 0,6 \% y del 1,8 \% en el caso de prevalencia de trastornos de la conducta (incluyendo hiperactividad).

Situándonos al comienzo del recorrido histórico, la introducción del término diagnóstico dual, con esta acepción, tiene lugar en la década de los noventa del siglo 
APROXIMACIÓN AL ESTUDIO DE LAS DIFERENCIAS INDIVIDUALES Y AL DIAGNÓSTICO DE PATOLOGÍA DUAL DESDE LA PERSPECTIVA PSICOLÓGICA EN NIÑOS Y ADOLESCENTES CON SÍNDROME DE DOWN G. DELGADO-PARDO

pasado. Serán Lovell y Reiss (1993) los primeros en referirse a ello, mientras que en España se atribuye el mismo reconocimiento del término al doctor Novell a finales de la citada década (Hernández et al., 2011). Hasta entonces e incluso después, la posible aparición de trastorno psicológico y/o enfermedad mental, junto a la discapacidad intelectual, se ha interpretado generalmente como un correlato propio del síndrome y/o en relación directa con el grado de discapacidad intelectual, siendo esta circunstancia el principal impedimento para la falta de atención a la posible existencia del doble diagnóstico (Garvía, 2014).

A continuación, con la intención de dar un orden a la bibliografía revisada y siguiendo el planteamiento de objetivos propuestos, se expone una evolución temporal desde los primeros estudios disponibles y hasta la actualidad.

\subsection{Antecedentes del estudio del diagnóstico dual en niños y adolescentes con SD (1930-1999)}

Las investigaciones muestran que la primera constancia sobre la presencia de patología dual en el SD aparece en 1934 cuando Earl describe la observación de síntomas de catatonia presentes en varios casos de personas con SD institucionalizadas. Este autor concluía que, dado el número de casos observados, podría incluso tratarse de una tendencia en el SD. Años después, en 1946, Rollin se refería a la presencia de síntomas de esta misma enfermedad psiquiátrica en 28 personas con SD de entre un total de 73 evaluadas. Y en 1979, Wakabayashi, en un artículo encaminado a esclarecer los mecanismos etiológicos del autismo, se referiría a la posible asociación entre autismo y SD en niños, aunque entendiéndola como una asociación extraña y aislada. Su estudio se basaba en la observación de un solo caso con esta doble patología, por lo que continuaba confirmando el carácter predominantemente extrovertido y afable de los niños con $\mathrm{SD}$, que contrastaba con los resultados de estudios previos como el de Rollin (1946), que se refería a cómo prácticamente la mitad de los niños con SD evaluados $(42,4 \%)$ se mostraban introvertidos, desinteresados, apáticos e impasibles, o, igualmente, los datos hallados por Menolascino en 1965 que encontró como al menos un 12,8 \% de los evaluados presentaban síntomas de trastornos emocionales por lo que llegó a afirmar que quizá habría que tomar con cautela la idea generalizada del carácter amigable y simpático de las personas con SD. En los años sucesivos, continúan apareciendo otras publicaciones sobre la presencia de trastornos mentales en niños con SD, en las que se describe la aparición de problemas de conducta grave y algún caso de autismo, pero en las que aún se continúa insistiendo en que se trata de un fenómeno particular y aislado (Gath y Gumley, 1986; Rutter y Schopler, 1988; Bregman y Wolkmar, 1988).

Otros autores comenzaban a interesarse por diferentes aspectos relacionados con la comorbilidad, por ejemplo, Gillberg et al. (1986), quienes, tras realizar un estudio epidemiológico, confirmaron la imposibilidad de referirse de forma generalizada al estereotipo del buen carácter del SD dada la alta incidencia de autismo en dicho síndrome. Otro autor, Lund (1988), aludía, en este caso, a la morbilidad psiquiátrica en población adulta al encontrar, entre los 44 participantes adultos con SD evaluados, como 11 de 
ellos presentaban algún tipo de trastorno y, en concreto, 5 casos de autismo. Myers y Pueschel en 1991, con el interés de conocer la prevalencia y la naturaleza de los trastornos psiquiátricos en personas con $\mathrm{SD}$, diseñaron un estudio con una muestra amplia $(\mathrm{N}=497)$. Este grupo tan numeroso fue dividido en dos de manera que, del total, 261 participantes eran menores de 20 años. Entre estos, el porcentaje de incidencia de patología dual alcanzó el 22,1 \%, siendo los trastornos más frecuentes observados para este grupo de edad la presencia de comportamientos disruptivos, trastornos de ansiedad y comportamientos repetitivos. Por su parte, Turk (1992) llevó a cabo un estudio de contraste, entre dos grupos de niños con SD y X frágil. En el caso del SD obtuvo una prevalencia de presencia de autismo del $9 \%$, aunque el propio autor admitió que los datos debían ser tomados con cautela, en relación con diferentes limitaciones metodológicas del estudio (no haber incluido a ninguna niña, el rango de edad estudiado, los instrumentos utilizados, los informantes y los criterios de diagnóstico empleados).

Junto a los estudios sobre incidencia y prevalencia, otra de las demandas más destacadas de la investigación del diagnóstico dual se refería a la necesidad de poder realizar estudios sistemáticos en los que evaluar los síntomas observables de manera acertada. Los autores insistían en este aspecto (Ghaziuddin et al., 1992; Ghaziuddin, 1997) al observar que era posible que, además de autismo, otros problemas de conducta pudieran superponerse, dificultando aún más el diagnóstico de comorbilidad. Con una muestra reducida de participantes, Howlin et al. (1995) presentaron cuatro casos de autismo junto a SD; cada uno de los evaluados cumplía criterios suficientes para este diagnóstico, pero a pesar de ello en cada caso los padres informaron acerca de las dificultades encontradas para poder obtenerlo. Entre esas dificultades se destacó el hecho de que los síntomas de los niños se atribuyeran a sus retrasos cognitivos, a pesar de que su comportamiento y su progreso general diferían de los de otros niños con SD en muchos aspectos importantes. Kent et al. (1999) se plantearon como objetivo conocer la prevalencia del autismo y TEA en una muestra de 58 menores con SD de los que 33 completaron la evaluación y 4 recibieron diagnóstico, el porcentaje de comorbilidad hallado fue del $7 \%$. En este caso, igualmente, los padres de los menores con antelación a la evaluación habían sugerido que sus hijos mostraban un comportamiento diferente al esperado en un niño con SD “estereotipo", sin embargo y del mismo modo que en el estudio anterior, de nuevo se atribuyeron estos síntomas observados a un mayor grado de discapacidad intelectual.

\subsection{Etapa intermedia y de desarrollo del estudio del diagnóstico dual en niños y ado- lescentes con SD (2000-2010)}

Este segundo periodo temporal ya parte de la aceptación del doble diagnóstico y como se ha podido observar, por el periodo anterior, al referirnos a población infantil y adolescente con SD será la sintomatología autista la que prevalezca por su incidencia a estas edades. Por otro lado, síntomas relacionados con el TDAH, los trastornos de atención y, conforme avanza la edad, los trastornos de conducta y/o los trastornos emocionales empiezan a aparecer en las publicaciones de manera, también, frecuente. 
APROXIMACIÓN AL ESTUDIO DE LAS DIFERENCIAS INDIVIDUALES Y AL DIAGNÓSTICO DE PATOLOGÍA DUAL DESDE LA PERSPECTIVA PSICOLÓGICA EN NIÑOS Y ADOLESCENTES CON SÍNDROME DE DOWN G. DELGADO-PARDO

En 1999, George Capone publica un monográfico dedicado a la presentación conjunta del SD junto al TEA, donde intenta esclarecer la descripción de los síntomas posibles, exponer las dificultades del diagnóstico y con el que solicita la necesidad de poder contar con profesionales (equipo de salud, terapeutas y educadores) cualificados, con capacidad para poder dar respuesta a esta demanda. Se trata de una publicación destinada, sobre todo, a las familias con la pretensión de acercarles y familiarizarles normalizando la existencia de este doble diagnóstico. Años después, Capone (2007) publica un trabajo de revisión donde ya en esta ocasión hace hincapié en la descripción de los trastornos neuroconductuales que pueden observarse junto al SD. Incide en el dato de las altas cifras de prevalencia sobre la presencia de comorbilidad, que se sitúan entre el $18 \%$ y $38 \%$, e insiste en la falta de instrumentos de evaluación específicos y en la consecuente escasez de diagnósticos acertados. Destacan entre los trastornos más frecuentes a estas edades tempranas el TDAH, el trastorno de conducta y el trastorno negativista desafiante y el TEA. En esta publicación, el autor presenta y utiliza la lista de verificación de comportamiento aberrante (ABC) (Aman et al., 1985), a la que se refiere como un instrumento específico para esta población, que parece estar proporcionando datos útiles sobre la detección del espectro autista en el SD.

Esta escala de verificación de comportamiento aberrante (ABC) se había utilizado con anterioridad por Capone como un instrumento apropiado para comprobar los síntomas cognitivos y conductuales del SD junto al TEA (Capone et al., 2005). Se pretendía que se utilizara como instrumento de diagnóstico, aunque en principio solo fuera aplicable al ámbito de investigación. De los resultados de su administración para esta población se obtuvo una buena correlación entre los criterios diagnósticos del DSM-IV para el autismo y las puntuaciones obtenidas por los participantes evaluados en las subescalas del instrumento, por lo que se reconocía su viabilidad para la evaluación del fenotipo neuroconductual de niños con trisomía 21 y TEA. En 2007 (Carter et al., 2007) volvieron a probar este instrumento con el interés de consolidar los resultados ya obtenidos, evaluando a una muestra de menores con SD utilizando esta escala (ABC), junto a la lista de verificación de comportamiento del autismo, por lo que otro objetivo más específico de este trabajo consistía en poder examinar qué comportamientos característicos distinguían el fenotipo conductual del SD, junto al autismo, de otros trastornos de la conducta en el SD. Los hallazgos encontrados pusieron de relieve que el diagnóstico dual SD + TEA representaba un conjunto de comportamientos diferentes, marcados por estereotipias características, ansiedad y retraimiento social. Tiempo después, y ante un cierto rechazo sobre la validez de los criterios diagnósticos del DSM para la valoración del TEA, ante la prevalencia de deterioro cognitivo en esta población, Ji et al. (2011) volvieron a administrar la lista de verificación de comportamiento aberrante $(\mathrm{ABC})$ para validar estos criterios. Del análisis final y considerando que esta escala había sido diseñada específicamente para personas con discapacidad intelectual severa, se pudo demostrar que los criterios para el diagnóstico de espectro autista basados en el DSM eran aplicables a las personas con SD. Entre los hallazgos obtenidos se identificaron dos grupos de menores que mostraban sintomatología específica y diferenciada (SD + TEA), de modo que se respaldaba la idea de la existencia de al menos dos subtipos de espectro autista en el 
SD; estos se distinguían con facilidad del grupo con SD y de aquellos que presentaban otros comportamientos desadaptativos.

Por otro lado, Elisabeth Dykens había estado participando en la publicación de trabajos previos sobre la conducta desadaptativa en el SD, con la intención de buscar explicaciones y/o justificaciones a estos comportamientos en relación con diferentes variables como la edad y sexo del menor, nivel de discapacidad intelectual e, incluso, el tipo de evaluación realizada. Tras estos trabajos previos y ante la escasez de investigación, en 2007 la autora publica un trabajo de revisión sobre la prevalencia de los trastornos del comportamiento y emocionales en las personas con SD (distinguiéndolos por periodos evolutivos). De manera que destaca entre sus hallazgos que no son tan frecuentes los problemas graves de conducta en los niños, pero considera los datos emergentes sobre autismo y resalta la falta de investigación sobre población adolescente. En el caso de los adultos, se refiere a la depresión y al alzhéimer en el caso de adultos mayores. La revisión termina con tres propuestas o enfoques necesarios sobre los que investigar al respecto. El primero, la necesidad de conectar el estado psicológico y los diagnósticos a lo largo de los periodos evolutivos y de desarrollo, incluyendo la adolescencia y considerando aspectos asociados, tales como sociabilidad, ansiedad y atención. En segundo lugar, la necesidad de prestar atención a los factores de riesgo biopsicosocial que puedan influir sobre el riesgo de psicopatología. Y, por último, destaca la necesidad de identificar los tratamientos más ajustados para estas personas.

En este punto, uno de los trabajos más completos de revisión hasta el momento lo publica Reilly (2009), que realiza un recorrido por la evolución de la aparición conjunta del SD junto al TEA, partiendo de las primeras publicaciones sobre la falta de reconocimiento de este doble diagnóstico hasta llegar a la situación actual. Se examinan las cifras de prevalencia y se explora la manifestación del TEA en el SD en relación con la expresión de síntomas identificables; e, igualmente, se revisan estudios de caso donde se investigan los instrumentos utilizados para su detección y diagnóstico. Por otro lado, se tienen en cuenta otras posibles variables intervinientes, tales como el nivel de funcionamiento cognitivo, factores médicos, edad, género, así como la influencia de posibles antecedentes familiares. Entre las conclusiones más destacadas, se deja en abierto la posibilidad de que el trastorno dual, SD y TEA, constituya un grupo cualitativamente diferente o se trate de un continuo entre SD y/o TEA. Siendo esta la clave fundamental de la investigación futura para la selección y/o creación de instrumentos de evaluación y para poder comprender el diagnóstico, así como poder realizar diagnósticos precoces y en cuanto a la elección adecuada de intervenciones multimodales ajustadas.

\section{3. Última década y actualidad (2010-2020)}

En este último periodo, la producción científica es más rigurosa y más continua. Se comprueba cómo empieza a haber un reconocimiento más extendido sobre la aceptación del doble diagnóstico, que no se restringe únicamente al ámbito de la investigación, aunque llama la atención cómo sigue habiendo excepciones. Por ejemplo, 
Krieger et al. (2014) presentaron una revisión sistemática de estudios realizada en Francia, pero cuyos resultados se referían a lo publicado en el ámbito anglosajón, en los 15 años anteriores, por falta de investigación y datos propios.

A continuación, en el cuadro que aparece se muestra una relación de aquellas investigaciones más representativas sobre patología dual en el menor con SD, en las que se muestra el avance de los intereses en estos últimos 10 años hasta llegar a la actualidad:

\begin{tabular}{|l|l|l|l|l|}
\hline \multicolumn{5}{|c|}{ TABLA 1. Investigaciones relacionadas con el estudio del diagnóstico dual } \\
en el SD publicadas en la última década (2010-2020)
\end{tabular}

Ediciones Universidad de Salamanca / CC BY-NC-ND

Siglo Cero, vol. 52 (4), 2021, octubre-diciembre, pp. 131-154 


\begin{tabular}{|c|c|c|c|c|}
\hline $\begin{array}{l}\text { Krieger, Lancéart, } \\
\text { Nader-Grosbois } \\
\text { y Adrien (2014) }\end{array}$ & FRANCIA & $\begin{array}{l}\text { Revisión bibliográfica } \\
\text { sobre diagnóstico dual } \\
\text { (SD + TEA) publicada } \\
\text { en el ámbito anglosa- } \\
\text { jón en los últimos } 15 \\
\text { años. }\end{array}$ & $\begin{array}{l}\text { Reforzar el conocimiento y las } \\
\text { actitudes de los profesionales } \\
\text { ante el diagnóstico dual. } \\
\text { Detectar patología dual SD + } \\
\text { autismo para una intervención } \\
\text { temprana. }\end{array}$ & $\begin{array}{l}\text { Se observa la aparición de signos clíni- } \\
\text { cos de autismo en niños SD. } \\
\text { Es necesaria la aplicación de herra- } \\
\text { mientas de diagnóstico específicas y } \\
\text { sensibles para esta población. } \\
\text { Necesaria la intervención temprana } \\
\text { con estos niños cuyas necesidades di- } \\
\text { fieren de aquellos SD sin autismo. }\end{array}$ \\
\hline $\begin{array}{l}\text { Ghaziuddin, } \\
\text { Nassiri y Miles } \\
(2015)\end{array}$ & EE. UU. & $\begin{array}{l}4 \text { casos de adolescentes } \\
\text { SD con síntomas de } \\
\text { regresión y deterioro } \\
\text { en diversas áreas (mo- } \\
\text { tora, del habla, com- } \\
\text { portamiento, estado de } \\
\text { ánimo y habilidades de } \\
\text { la vida diaria). }\end{array}$ & $\begin{array}{l}\text { Advertir a los profesionales } \\
\text { sobre la aparición de catatonia } \\
\text { en jóvenes SD y fomentar la } \\
\text { investigación sobre la detección } \\
\text { precoz de síntomas regresivos. }\end{array}$ & $\begin{array}{l}\text { Se evidencia la aparición repentina y } \\
\text { progresiva de regresión en adolescen- } \\
\text { tes y jóvenes adultos SD. La falta de } \\
\text { familiarización con el diagnóstico de } \\
\text { catatonia en el SD favorece diagnós- } \\
\text { ticos erróneos y años de morbilidad. }\end{array}$ \\
\hline $\begin{array}{l}\text { Channell et al., } \\
\text { (2015) }\end{array}$ & EE. UU. & $\mathrm{N}=46$ (10-21 años). & $\begin{array}{l}\text { Examinar aparición conjunta } \\
\text { de TEA + SD, en una muestra } \\
\text { de personas SD sin diagnóstico } \\
\text { previo de autismo. Evaluados a } \\
\text { través de la Escala de Responsi- } \\
\text { vidad Social (SRS). }\end{array}$ & $\begin{array}{l}\text { Puntajes elevados de sintomatología } \\
\text { TEA en el SRS indican la necesidad } \\
\text { de desarrollar normas específicas de } \\
\text { diagnóstico de este trastorno para el } \\
\text { SD y evitar los falsos positivos. }\end{array}$ \\
\hline $\begin{array}{l}\text { Naerland et al., } \\
\text { (2016) }\end{array}$ & $\begin{array}{l}\text { NORUEGA, } \\
\text { REINO } \\
\text { UNIDO y } \\
\text { AUSTRALIA }\end{array}$ & $\mathrm{N}=674$ (4-18 años). & $\begin{array}{l}\text { Estudio multicentro sobre } \\
\text { prevalencia de patología dual } \\
\text { (autismo, problemas emocio- } \\
\text { nales, conductuales y TDAH) } \\
\text { y su relación con edad, sexo y } \\
\text { grado de discapacidad intelec- } \\
\text { tual, en una muestra amplia de } \\
\text { menores SD. }\end{array}$ & $\begin{array}{l}\text { Tasas elevadas de presencia de difi- } \\
\text { cultades de comportamiento ( } 48 \% \text { ), } \\
\text { síntomas de hiperactividad y falta de } \\
\text { atención }(34 \%) \text { y sintomatología au- } \\
\text { tista }(17 \% \text { ) entre niños SD. } \\
\text { Se destaca la necesidad de identifica- } \\
\text { ción de esta sintomatología por parte } \\
\text { de los profesionales (médicos, educa- } \\
\text { dores y psicólogos). } \\
\text { Sin resultados relevantes en relación } \\
\text { con otras variables estudiadas (edad, } \\
\text { sexo y grado de discapacidad). }\end{array}$ \\
\hline Fujino (2017) & JAPÓN & $\begin{array}{l}\text { Estudio de interven- } \\
\text { ción psicosocial sobre } \\
\text { dos casos de adultos } \\
\text { jóvenes SD. }\end{array}$ & $\begin{array}{l}\text { Implementar un programa } \\
\text { de intervención psicosocial, } \\
\text { Dohsa-hou, para el manejo de } \\
\text { sintomatología psicológica y } \\
\text { trastornos emocionales en per- } \\
\text { sonas jóvenes SD. }\end{array}$ & $\begin{array}{l}\text { Se obtuvo mejoría en ambos casos de } \\
\text { estudio, siendo mejores los resultados } \\
\text { en uno de los dos pacientes. Estas di- } \\
\text { ferencias en los resultados tienen rela- } \\
\text { ción con el nivel de adaptación de los } \\
\text { pacientes al tratamiento y la gravedad } \\
\text { previa de los síntomas psicológicos } \\
\text { manifiestos. }\end{array}$ \\
\hline $\begin{array}{l}\text { Marino et al. } \\
\text { (2019) }\end{array}$ & ITALIA & $\mathrm{N}=97$ (1-18 años). & $\begin{array}{l}\text { Identificar sintomatología psi- } \\
\text { copatológica y/o autismo en } \\
\text { niños y adolescentes SD a partir } \\
\text { del Cuestionario sobre el Com- } \\
\text { portamiento de niños y adoles- } \\
\text { centes (CBCL) y la escala de } \\
\text { calificación (CARS-T) para la } \\
\text { evaluación de autismo infantil. }\end{array}$ & $\begin{array}{l}\text { Alta incidencia del riesgo psicopatoló- } \\
\text { gico en la muestra de estudio ( } 90 \% \text { ) } \\
\text { y presencia de características autistas } \\
(7 \%) \text {. } \\
\text { Se destaca la importancia del uso de } \\
\text { herramientas de detección específi- } \\
\text { cas que no enmascaren los síntomas. } \\
\text { El riesgo de psicopatología es inde- } \\
\text { pendiente del grado de discapacidad } \\
\text { intelectual. }\end{array}$ \\
\hline
\end{tabular}

Ediciones Universidad de Salamanca / CC BY-NC-ND

Siglo Cero, vol. 52 (4), 2021, octubre-diciembre, pp. 131-154 
APROXIMACIÓN AL ESTUDIO DE LAS DIFERENCIAS INDIVIDUALES Y AL DIAGNÓSTICO DE PATOLOGÍA DUAL DESDE LA PERSPECTIVA PSICOLÓGICA EN NIÑOS Y ADOLESCENTES CON SÍNDROME DE DOWN G. DELGADO-PARDO

\begin{tabular}{|c|c|c|c|c|}
\hline $\begin{array}{l}\text { Hamner et al. } \\
(2020)\end{array}$ & EE. UU. & $\begin{array}{l}\mathrm{N}=173(5 \text { meses- } 6 \\
\text { años). } \\
\text { Tres grupos: } \\
\quad \text { - SD. } \\
\quad \text { - TEA. } \\
\quad \text { - SD + TEA. }\end{array}$ & $\begin{array}{l}\text { Evaluar la prevalencia de TEA } \\
+ \text { SD. } \\
\text { Describir el fenotipo conduc- } \\
\text { tual del trastorno dual, SD + } \\
\text { TEA, en relación con el SD y al } \\
\text { TEA de manera aislada. }\end{array}$ & $\begin{array}{l}\text { Los menores SD + TEA muestran } \\
\text { mayor deterioro en relación TEA-no } \\
\text { SD y SD-no TEA y menor gravedad } \\
\text { de los síntomas autistas en relación } \\
\text { con el TEA. } \\
\text { Existe un perfil conductual específico } \\
\text { SD + TEA. } \\
\text { Necesidad de diagnóstico precoz e } \\
\text { intervención temprana. }\end{array}$ \\
\hline $\begin{array}{l}\text { Ortiz Videla et al. } \\
\text { (2017) }\end{array}$ & ESPAÑA & 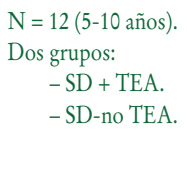 & $\begin{array}{l}\text { Estudio observacional y retros- } \\
\text { pectivo para identificar señales } \\
\text { precoces de TEA en niños SD } \\
\text { pequeños ( } 0-4 \text { años). }\end{array}$ & $\begin{array}{l}\text { Identificación de señales precoces de } \\
\text { alarma sobre el diagnóstico dual SD } \\
\text { + TEA del tipo "no imita", "no inte- } \\
\text { gra la mirada", "falta de interés por el } \\
\text { otro", "falta de atención compartida", } \\
\text { etc. }\end{array}$ \\
\hline
\end{tabular}

Al inicio de la década los estudios realizados, con muestras amplias, muestran datos sobre prevalencia donde continúa observándose la misma tendencia, cifras muy elevadas y muy variables. DiGuiseppi et al. (2010) se referían a un 18,2 \% de prevalencia de sintomatología TEA y del 6,4 \% de autismo en una muestra de 120 menores con SD (2-11 años). Ante estos datos y a pesar de que dos de los cuatro cuestionarios aplicados demostraron sensibilidad para la detección de sintomatología TEA en el SD, los autores reclamaban la creación de instrumentos con los que poder controlar de manera fiable los falsos positivos. En el caso del TDAH, Ekstein et al. (2011) indicaron un $44 \%$ de prevalencia de este trastorno en el SD, en una muestra con 41 participantes, sin que se obtuvieran datos sobre la influencia de un mayor grado de discapacidad intelectual en relación con la aparición del trastorno dual. Naerland et al. (2016) (674 evaluados) confirmaban tasas relativamente altas sobre la presencia de dificultades del comportamiento y la observación de síntomas de hiperactividad, falta de atención y autismo en niños con SD. Tampoco se obtuvieron datos sobre la asociación de estos síntomas junto a otras variables estudiadas (edad, sexo y grado de discapacidad). En nuestro ámbito, Ortiz et al. (2017) se referían a la prevalencia de comorbilidad entre el SD y el TEA, por lo que presentaron un estudio retrospectivo para la identificación precoz de signos de alarma de la doble patología. Se trata de un estudio pionero en nuestro país y, a pesar de sus limitaciones metodológicas, constata la necesidad de considerar el doble diagnóstico en la población con SD, con una aparición tardía de los síntomas y donde no se cuenta con protocolos de evaluación ni con instrumentos específicos de diagnóstico. Warner et al. (2017) informaron sobre una muestra de 499 personas con SD, donde al menos 183 del total cumplieron criterios para el diagnóstico dual, situándose el porcentaje de prevalencia entre el $16 \%$ y el $18 \%$, muy por encima de lo esperado en población general. Los autores volvían a insistir sobre la necesidad de instrumentación específica que facilitase describir perfiles de comportamiento y sintomatología emocional distinta, con relación al SD y, también, al TEA.

Previo a ello, los autores coincidían en el perfil diferenciador de los menores de doble diagnóstico situándose en diferentes posicionamientos, en unos casos, acercán- 
APROXIMACIÓN AL ESTUDIO DE LAS DIFERENCIAS INDIVIDUALES Y AL DIAGNÓSTICO DE PATOLOGÍA DUAL DESDE LA PERSPECTIVA PSICOLÓGICA EN NIÑOS Y ADOLESCENTES CON SÍNDROME DE DOWN G. DELGADO-PARDO

dolos del lado de los autistas, de manera que estos menores mostraban más estereotipias, lenguaje repetitivo, hiperactividad y autolesiones, que en relación con los "sólo" con SD (Moss et al., 2012). Mientras que otros autores se referían a un parecido más cercano en cuanto a comportamiento adaptativo entre los niños con SD + autismo y los “solo" con SD, que en relación con los “solo” con autismo (Dressler et al., 2011). Sin embargo, los datos más recientes sostienen que los menores diagnosticados con patología dual son diferentes a cualquier forma de presentación de los dos trastornos, SD y TEA, de forma aislada (Godfrey et al., 2019) y por ello, en la actualidad, los autores intentan centrarse en poder describir fenotipos de desarrollo y de comportamiento específicos de este grupo, que posibiliten el diagnóstico precoz y el diseño de intervenciones terapéuticas orientadas al reforzamiento de su funcionamiento social, que podrían resultar muy beneficiosas para estos menores (Hamner et al., 2020).

Los resultados sobre la intervención psicológica en la patología dual, hasta el momento, son muy pocos, aunque los primeros datos parecen indicar que son satisfactorios (Ortiz, 2012). Stein et al. (2013) presentan un solo caso de intervención con una chica de 13 años con SD. Se trata de un estudio de caso en el que la chica presentaba una regresión cognitiva y del desarrollo, hasta el punto de manifestar pérdidas en el lenguaje, habilidades sociales y del uso del baño. Tras el descarte de posibles diagnósticos médicos y en vista de la ocurrencia de una serie de acontecimientos psicosociales (mudanza, inicio de menstruación, cambio de aula y compañeros de clase) se procedió con una evaluación integral, psicológica y psiquiátrica, por la que se diagnosticó una depresión reactiva. De la evaluación neuropsicológica se observó disminución en la motivación y el rendimiento, con una reacción de estrés como factor causal potencial. El tratamiento llevado a cabo incluyó psicofármacos, terapia médica y un programa de apoyo psicosocial dirigido para la adaptación a los cambios ambientales. Con este enfoque de tratamiento multimodal se logró una mejoría observable en los síntomas, lográndose que la chica se recuperarse hasta niveles de funcionamiento previos al episodio. Fujino (2017) presentó los hallazgos obtenidos a partir de la intervención con un programa psicosocial conocido como Dohsa-hou, en dos casos de aparición de comorbilidad. Dohsa-hou es un tipo de tratamiento, basado en un enfoque psicosocial, utilizado en la práctica clínica, principalmente, en Japón y los países de Asia Oriental. Al término del tratamiento los participantes mostraron una mejoría en conductas desadaptativas y en cuanto a problemas de internalización, sin embargo, los autores refirieron observar cómo la mejoría diferencial en cada caso pudo estar influenciada por la gravedad inicial de los síntomas. Finalmente, se concluyó a favor de la eficacia de Dohsa-hou para mejorar la adaptación a sintomatología internalizante en adultos con SD. Davis et al. (2018), a pesar de la escasa investigación sobre el tratamiento de la patología dual, SD y TEA, presentaron los resultados sobre la efectividad de un programa de instrucción directa mediada por pares para el aprendizaje de habilidades sociales en esta población. El objetivo el programa consistía en aumentar las habilidades sociales de estos menores (SD + TEA) y que aprendieran a generalizarlas a diferentes entornos. Los resultados, igualmente, resultaron satisfactorios al mostrar la eficacia de este tipo de intervención para esta población. 
Por último, otro síndrome neuropsiquiátrico al que referirse por su relevancia y el desarrollo reciente de su estudio, por aparecer junto al SD, por su incidencia y desconocimiento, es la catatonia. De hecho, su prevalencia en el SD continúa siendo desconocida, a pesar de que no se haya dejado de investigar desde aquellos primeros estudios citados de principios del siglo XX. Quizá la justificación de este desconocimiento se deba a que los síntomas regresivos que con más frecuencia se identifican en población adolescente o adultos jóvenes con SD siguen confundiéndose con depresión o con signos de un inicio temprano de alzhéimer. En 2015 Ghaziuddin, Nassiri y Miles identificaban y describían 4 casos de catatonia en 4 jóvenes (15-18 años). Estos autores, en las conclusiones de su trabajo, explicaban que posiblemente la causa del deterioro inexplicable de estos adolescentes podía deberse a este trastorno que suele pasar desapercibido por desconocimiento, incluso, por parte de los especialistas insuficientemente familiarizados con su diagnóstico. Worley et al. (2015) presentaron, el mismo año, un análisis de 11 casos observados en un periodo de seguimiento de 10 años (2002-2012). En este caso, para poder diagnosticarlos, los menores debían presentar una regresión autista acorde a criterios diagnósticos del DSM-IV R, un deterioro cognitivo significativo y una edad superior a los 5 años, aunque la edad media de aparición de los síntomas, para este grupo, fue de 11,4 años. Estos resultados coinciden con otros aportados, que sitúan la edad de inicio de la regresión del desarrollo en la adolescencia, caracterizándose por una pérdida del funcionamiento adaptativo, cognitivo y social adquirido previamente por los menores con SD (Rosso et al., 2020). Otros síntomas que aparecerían relacionados incluirían inestabilidad del estado de ánimo, disminución de la participación en actividades de la vida diaria, insomnio, retraimiento social, mutismo y catatonia. Suele iniciarse con una fase aguda que es seguida de una fase de cronificación progresiva de la que puede que no haya reversibilidad. Santoro et al. (2020) comenzaron un estudio en 2017, en colaboración con un consorcio de centros para personas con SD; pretendían recopilar datos para poder realizar un seguimiento del diagnóstico. Identificaron 35 casos de jóvenes con SD (17,5 años de media) que habían sufrido una regresión inexplicable. Los síntomas y el diagnóstico coincidían con los propuestos para este síndrome por lo que se demandaba la necesidad de poder definir clínicamente el síndrome e investigarlo, para poder identificar los mecanismos subyacentes. A su vez, los autores en esta publicación se refieren a que, aunque el tipo de regresión parece distinguirse de otros diagnósticos que cursan con pérdidas de habilidades en el SD, aún no existe un acuerdo común sobre la identificación del diagnóstico con un término proponiendo, en su caso, el de "unexplained regresión in Down síndrome”. En España se ha propuesto identificarlo como regresión de origen desconocido en el síndrome de Down (Flórez y Ortega, 2020).

\section{Conclusiones}

El objetivo principal de la revisión y del análisis del articulado expuesto consiste en, poner de relieve la necesidad de abordar el estudio del SD desde la perspectiva de las diferencias individuales $\mathrm{y}$, por consiguiente, poder realizar un acercamiento cer- 
tero hacia la descripción de la patología dual en dicha población. Esto es, conocer los síntomas y/o trastornos psicológicos que con más frecuencia suelen aparecer junto al SD a edades tempranas, en niños y adolescentes. Siendo el propósito final acercar los avances observados en torno al tema de investigación a las familias de los menores y favorecer su familiarización y adaptación ante la posibilidad del doble diagnóstico y, en el caso de los profesionales, reforzar este conocimiento ante la demanda de tener que realizar diagnósticos precoces y ajustados, que faciliten el diseño de programas de intervención multimodales que incluyan tratamientos psicológicos que resulten adecuados a las necesidades individuales y específicas de estos menores.

$\mathrm{Y}$ es que, desde un punto de vista psicológico, definir los rasgos de las personas con SD a partir de estereotipos resulta demasiado simplista. El SD continúa siendo el motivo más frecuente de discapacidad intelectual asociada a anomalía genética, que conlleva una afectación en el desarrollo físico y psicológico que dará lugar a un fenotipo característico que, sin embargo, variará considerablemente con relación al propio desarrollo madurativo, la gravedad de las discapacidades específicas del menor y en función de la influencia de su entorno social (Oxelgren et al., 2017; Troncoso, 2019). Junto a ello es sabido, y así lo indica la bibliografía revisada, cómo la aparición de síntomas psicológicos (y/o psiquiátricos) junto a la discapacidad intelectual es más frecuente que en población general, lo que también sucede, aunque en menor medida, en las personas con SD. Bajo estas circunstancias, se incide en la necesidad de desechar concepciones que se refieren al enmascaramiento de los síntomas psicológicos como propios de la discapacidad y superar la idea clásica acerca de las dificultades cognitivas y de comunicación de las personas con SD para poder manifestar dichos síntomas puesto que no favorecen la atención individualizada necesaria (Garvía, 2014; Garvía, 2017).

En el desarrollo del presente estudio, y partiendo de la postura centrada en las diferencias individuales, se realiza una exposición de aquellas investigaciones más relevantes organizadas temporalmente en tres momentos diferenciados. En el primero de ellos, se destaca la prematuridad de los primeros estudios que se sitúan a mediados del siglo pasado y que se refieren casi en su mayoría a la pronta observación de síntomas de autismo junto al SD (Reilly, 2009). A partir de estos primeros estudios, se confirma la obtención de datos más que suficientes sobre la incidencia y prevalencia de sintomatología comórbida que justifican la continuación de esta línea de investigación, haciendo especial hincapié en la realización de estudios con mayor rigor metodológico. Estos trabajos pioneros dan muestra de cómo la idea prevalente sobre el estereotipo de sociabilidad y su relación con la percepción de grupo homogéneo en el SD no favorecen el desarrollo de los aspectos sociales y cognitivos de esta población. Hasta el momento, los síntomas que no se consideran propios del síndrome se asocian en su mayoría al grado de discapacidad intelectual presente en los menores, hecho que queda descartado tal y como se refleja en los estudios analizados sobre la relación entre los síntomas observados y el nivel de inteligencia de los menores (Yahia et al., 2014; Naerland et al., 2016; Marino et al., 2019).

En un segundo periodo temporal, los artículos revisados se diversifican en cuanto a temas de estudio. Se destaca la necesidad de creación de instrumentos específicos 
APROXIMACIÓN AL ESTUDIO DE LAS DIFERENCIAS INDIVIDUALES Y AL DIAGNÓSTICO DE PATOLOGÍA DUAL DESDE LA PERSPECTIVA PSICOLÓGICA EN NIÑOS Y ADOLESCENTES CON SÍNDROME DE DOWN G. DELGADO-PARDO

de evaluación que faciliten realizar trabajos más rigurosos que incluyan: mecanismos eficaces de cribado de síntomas (Capone et al., 2005); la realización de estudios con muestras amplias y de contrastes poblacionales, así como la evaluación de la población con SD de diferentes edades (y/o momentos evolutivos) y, por supuesto, que se incluya en las muestras a niñas. Aparecen, igualmente, las primeras revisiones sobre el tema en cuestión donde se incide en la necesidad de prestar atención al diagnóstico de patología dual en el SD, tratando de explorar cuáles son las patologías psicológicas más frecuentes (TEA, trastorno de conducta, trastorno por déficit de atención con hiperactividad, depresión en los adolescentes, etc.) (Capone, 2007; Dykens, 2007; Reilly, 2009).

El tercer momento temporal coincide con el periodo actual. Se obtienen diferentes referencias sobre estudios rigurosos realizados con muestras amplias y de comparación poblacional que tratan de poner de relieve aspectos relacionados con la expresión de los síntomas observados en la patología dual en el SD, donde se concluye reforzándose la idea sobre la prefiguración de un perfil comportamental en estos menores diferente a lo observado en la presentación individual de las comorbilidades, esto es SD y/o TEA (DiGuiseppi et al., 2010; Dressler et al., 2011; Naerland et al., 2016), con lo cual se destaca la necesidad de la detección y la exploración precoces de la sintomatología para poder prestar una atención ajustada e individualizada.

En relación con la intervención desde la perspectiva psicológica en la patología dual en el SD, se destaca la falta de datos al respecto, indicándose que son aún pocos los estudios sobre tratamientos, por lo que se incide en la necesidad de realizar más investigación sobre este tema, sobre todo, porque los datos disponibles sobre la eficacia de los tratamientos ofrecen resultados positivos y similares a los observados en población general (Ortiz, 2012). Igualmente se refiere una creciente demanda sobre la necesidad de diseñar intervenciones terapéuticas multimodales, que incluyan tratamiento psicológico y que se ajusten a los perfiles específicos observados en estos menores (Moss et al., 2012; Dressler et al., 2011; Godfrey et al., 2019).

Se destaca la importancia de acercar la concepción del diagnóstico dual a las familias puesto que hasta el momento se encuentran posturas ambivalentes de aceptación y de rechazo, esta última sobre todo por falta de información y conocimientos. Se debe permitir y reforzar, por tanto, una postura de cambio y afrontamiento en los padres de estos menores que les impulse a solicitar la ayuda necesaria (Patterson, 1999; Hartway, 2016); se debe reforzar, igualmente, la misma postura en los educadores y terapeutas hacia la aceptación de la existencia del diagnóstico dual y la necesidad de formación especializada tanto en la identificación de sintomatología como para la posterior atención e intervención ya que se tiene constancia de que aún hoy encuentran dificultades para identificar e interpretar este tipo de síntomas (Ortiz Videla et al., 2017).

Finalmente, y de cara a la realización de posibles trabajos futuros, sería de interés poder realizar la replicación de estudios sobre la evaluación de la presencia de sintomatología psicológica en niños y adolescentes con síndrome de Down en nuestro ámbito cercano; así como la posibilidad de diseñar programas de intervención sobre estos datos que incluyan tratamiento psicológico dirigido a procurar una mejor adap- 
APROXIMACIÓN AL ESTUDIO DE LAS DIFERENCIAS INDIVIDUALES Y AL DIAGNÓSTICO DE PATOLOGÍA DUAL DESDE LA PERSPECTIVA PSICOLÓGICA EN NIÑOS Y ADOLESCENTES CON SÍNDROME DE DOWN G. DELGADO-PARDO

tación comportamental y emocional en estos menores que presenten patología dual en nuestro entorno.

Por último, añadir cómo con el desarrollo de este trabajo ha tomado relevancia otra posibilidad de investigación necesaria y en auge, que tiene que ver con el estudio de la presencia de catatonia y/o el trastorno desintegrativo en niños y adolescentes con SD. A partir del análisis de la bibliografía se ha comprobado que, si bien los primeros resultados sobre este tema de estudio vuelven a situarse bastante tiempo atrás (Ghaziuddin et al., 2015), es ahora, en la actualidad, cuando se empiezan a obtener datos significativos sobre su frecuencia y manifestación. A partir de los resultados de incidencia y dada la importancia de los síntomas observados, y dado el alcance de sus consecuencias para estos menores, se ha convertido en una prioridad necesaria conocer e identificar este trastorno para poder prestar atención y tratamiento de forma precoz e inmediata (Santoro et al., 2020).

\section{Referencias bibliográficas}

Akahoshi, K., Matsuda, H., Funahashi, M., Hanaoka, T. y Suzuki, Y. (2012). Acute neuropsychiatric disorders in adolescents and young adults with Down syndrome: Japanese case reports. Neuropsychiatric Disease and Treatment, 8, 339-345. https://doi:10.2147/ NDT.S32767

Aman, A., Sigh, N., Stewart, A. y Field, C. (1985). The aberrant behavior checktlist: a behavior rating scale for the assessment of treatment effects. American Journal of Mental Deficiency, 89, 485-491.

Arranz, P. (2002). Niños y jóvenes con sindrome de Down. Egido Editorial.

Basile, H. S. (2008). Retraso mental y genética. Síndrome de Down. Revista Argentina de Clínica Neuropsiquiátrica, 15(1), 9-23.

Bregman, J. D. y Volkmar, F. R. (1988). Autistic social dysfunction and Down syndrome. Journal of the American Academy of Child and Adolescent Psychiatry, 27, 440-441.

Campbell, J., Gilmore, L. y Cuskelly, M. (2003). Changing student teachers' attitudes towards disability and inclusion. Journal of Intellectual and Developmental Disability, 28(4), 369379. https://doi.org/10.1080/13668250310001616407

Candel, I. (1999). Programa de atención temprana. Intervención en niños con sindrome de



Capone, G. (1999). Down syndrome and autistic spectrum disorder: a look at what we know. Disability Solutions, 3, issues 5-6.

Capone, G., Goyal, P., Ares, W. y Lannigan, E. (2007). Trastornos neuroconductuales en niños, adolescentes y adultos jóvenes con síndrome de Down. Revista Síndrome de Down: Revista Española de Investigación e Información sobre el Sindrome de Down, 93, 52-61.

Capone, G. T., Grados, M. A., Kaufmann, W. E., Bernad-Ripoll, S. y Jewell, A. (2005). Down syndrome and comorbid autism-spectrum disorder: characterization using the aberrant behavior checklist. American Journal of Medical Genetics, 134(4), 373-380.

Carter, J. C., Capone, T. G., Gray, R. M., Cox, Ch. S. y Kaufmann, W. E. (2007). Autistic Spectrum Disorders in Down syndrome: further delineation and distinction from other behavioral abnormalities. American Journal of Medical Genetics, Part B (Neuropsychiatric Genetics) 144B, 87-94. 
APROXIMACIÓN AL ESTUDIO DE LAS DIFERENCIAS INDIVIDUALES Y AL DIAGNÓSTICO DE PATOLOGÍA DUAL DESDE LA PERSPECTIVA PSICOLÓGICA EN NIÑOS Y ADOLESCENTES CON SÍNDROME DE DOWN G. DELGADO-PARDO

Cebula, K. R., Moore, D. G. y Wishart, J. G. (2010). Social cognition in children with Down's syndrome: challenges to research and theory building. Journal of Intellectual Disability Research, 54. Part 2, 113-134. https://doi:10.1111/j.1365-2788.2009.01215.x

Channell, M. M., Phillips, B. A., Loveall, S. J., Conners, F. A., Bussanich, P. M. y KlinGER, L. G. (2015). Patterns of autism spectrum symptomatology in individuals with Down syndrome without comorbid autism spectrum disorder. Journal of Neurodevelopmental Disorders, 7(1), art. N. ${ }^{\circ}$ 5. https://doi:10.1186/1866-1955-7-5

Collacott, R. A., Cooper, S., Branford, D. y Mcgrother, C. (1998). Behaviour phenotype for Down's syndrome. The British Journal of Psychiatry, 172(1), 85-89. https://dx.doi. org/10.1192/bjp.172.1.85

Coppus, A. (2017). Comparing generational differences in persons with Down syndrome. Journal of Policy and Practice in Intellectual Disabilities, 14(2), 118-123. https://doi. org/10.1111/jppi.12214

Davis, M. A. C., Spriggs, A., Rodgers, A. y Campbell, J. (2018). The effects of a peer-delivered social skills intervention for adults with comorbid Down syndrome and Autism Spectrum Disorder. Journal of Autism and Developmental Disorders, 48(6), 1869-1885. https:// doi:10.1007/s10803-017-3437-1

Diguiseppi, C., Hepburn, S., Davis, J. M., Fidler, D. J., Hartway, S., Lee, N. R., Miller, L., RutTenber, M. y Robinson, C. (2010). Screening for Autism Spectrum Disorders in children with Down syndrome. Journal of Developmental and Behavioral Pediatrics, 31(3), 181-191. https://doi.org/10.1097/Dвр.0b013e3181d5aa6d

Dressler, A., Perelli, V., Bozza, M. y Bargagna, S. (2011). The autistic phenotype in Down syndrome: differences in adaptive behavior versus Down syndrome alone and autistic disorder alone. Functional Neurology, 26(3), 151-158.

Dykens, E. (1995). Measuring behavioural phenotypes: provocations from the "new genetics". American Journal on Mental Retardation, 99, 522-532.

Dykens, E. M. (2007). Psychiatric and behavioral disorders in persons with Down syndrome. Mental Retardation and Developmental Disabilities Research Reviews Journal, 13(3), 272278.

Dykens, E. M., Shah, B., Davis, B., Baker, C., Fife, T. y Fitzpatrick, J. (2015). Psychiatric disorders in adolescents and young adults with Down syndrome and other intellectual disabilities. Journal of Neurodevelopmental Disorders, 7(1), 9. https://doi:10.1186/s11689015-9101-1

EarL, C. J. C. (1934). The primitive catatonic psychosis of idiocy. British Journal of Medical Psychology, 14, 230-253. https://doi.org/10.1111/j.2044-8341.1934.tb01122.x

Ekstein, S., Glick, B., Weill, M., Kay, B. y Berger, I. (2011). Down syndrome and attentiondeficit/hyperactivity disorder (ADHD). Journal of Child Neurology, 26(10), 1290-1295. https://doi:10.1177/0883073811405201

FIDLER, D. J. (2005). The emerging Down syndrome behavior phenotype in early childhood: implications for practice. Infants and Young Children, 18(2), 86-103. https://dx.doi. org/10.1097/00001163-200504000-00003

FIDLER, D. J. (2006). The emergence of a syndrome-specific personality profile in young children with Down syndrome. Down's Syndrome Research and Practice, 10(2), 53-60. https:// doi:10.3104/reprints.305

Fidler, D. J., Barrett, K. C. y Most, D. E. (2005). Age-related differences in smiling and personality in Down syndrome. Journal of Developmental and Physical Disabilities, 17, 263-280. https://doi.org/10.1007/s10882-005-4384-x 
APROXIMACIÓN AL ESTUDIO DE LAS DIFERENCIAS INDIVIDUALES Y AL DIAGNÓSTICO DE PATOLOGÍA DUAL DESDE LA PERSPECTIVA PSICOLÓGICA EN NIÑOS Y ADOLESCENTES CON SÍNDROME DE DOWN G. DELGADO-PARDO

Fidler, D. J., Hepburn, S. y Rogers, S. (2006). Early learning and adaptive behaviour in toddlers with Down syndrome: evidence for an emerging behavioural phenotype? Down Syndrome Research Practice, 9(3), 37-44. https://doi:10.3104/reports.297

Flórez, J. (2005). Autismo y síndrome de Down. Revista Síndrome de Down, 22, 61-72.

Flórez, J. (2019). Diferencias individuales en el síndrome de Down. Revista Síndrome de Down, 36(II), 52-57.

Flórez, J. y Dierssen, M. (2006). La transcripción de genes en las personas con síndrome de Down: tan iguales y tan diferentes. Revista Sindrome de Down, 23, 78-83.

Flórez, J., Garvía, B. y Fernández-Olaria, R. (2015). Síndrome de Down: neurobiología, neuropsicología, salud mental. Bases para la intervención en el aprendizaje, la conducta y el bienestar mental. CEPE, Fundación Iberoamericana Down21.

Flórez, J. y Ortega, M. (2020). Regresión de origen desconocido en el sindrome de Down: una nueva entidad. Fundación Iberoamericana Down 21.

Flórez, J. y Ruiz, E. (2004). El síndrome de Down: aspectos biomédicos, psicológicos y educativos. Revista Virtual-Fundación Down 21.

FujINO, A. (2017). Psychological support for young adults with Down syndrome Dohsa-Hou Program for maladaptative behaviors and internalizing problems. Frontiers in Psychology, 1(8), 1504. https://doi:10.3389/fpsyg.2017.01504

Gallart i Valls, C. (2005). Psicoterapia individual para personas con síndrome de Down. Revista Médica Internacional sobre el Sindrome de Down, 9(3), 45-48. https://doi. org/10.1016/S1138-2074(05)70063-4

Garvía, B. (2014). Trastornos mentales en el síndrome de Down. Síndrome de Down: vida adulta, 17. https://www.sindromedownvidaadulta.org/revista-de-adultos-n-17-junio-2014/articulos-no17/trastornos-mentales-en-el-sindrome-de-down/

Garvía, B. (2017). Síndrome de Down y Trastorno del Espectro Autista. Revista Médica Internacional sobre el Sindrome de Down, 21(1), 1-2.

Gath, A. y Gumley, D. (1986). Behaviour problems in retarded children with special reference to Down's syndrome. The British Journal of Psychiatry, 149, 156-161. https://doi:10.1192/ bjp.149.2.156

GHAZIuddin, M. (1997). Autism in Down's syndrome: family history correlates. Journal of Intellectual Disability Research, 41, part. I, 87-91.

Ghaziuddin, M., Tsai, L. Y. y Ghaziuddin, N. (1992). Autism in Down's syndrome: presentation and diagnosis. Journal of Intellectual Disability Research, 36, 449-456.

Ghaziuddin, N., Nassiri, A. y Miles, J. H. (2015). Catatonia in Down syndrome: a treatable cause of regression. Neuropsychiatric Disease and Treatment, 11, 941-949. https:// doi:10.2147/NDT.S77307

Ghosh, M., Shah, A. H., Dhir, K. y Merchant, K. F. (2008). Behavior in children with Down syndrome. The Indian Journal of Pediatrics, 75(7), 685-689. https://doi:10.1007/s12098008-0129-z

Gibbs, M. V. y Thorpe, J. G. (1983). Personality stereotype of noninstitutionalized Down syndrome children. American Journal of Mental Deficiency, 87(6), 601-605.

Gillberg, C., Persson, E., Grufmann, N. y Themner, U. (1986). Psychiatric disorders in mildly and severely mentally retarded urban children and adolescents: epidemiological aspects. British Journal of Psychiatry, 149, 68-74. https://doi:10.1192/bjp.149.1.68

Glasson, E. J., Dye, D. E. y BitTles, A. H. (2014). The triple challenges associated with age-related comorbidities in Down syndrome. Journal of Intellectual Disability Research, 58(4), 393-398. https://doi:10.1111/jir.12026 
APROXIMACIÓN AL ESTUDIO DE LAS DIFERENCIAS INDIVIDUALES Y AL DIAGNÓSTICO DE PATOLOGÍA DUAL DESDE LA PERSPECTIVA PSICOLÓGICA EN NIÑOS Y ADOLESCENTES CON SÍNDROME DE DOWN G. DELGADO-PARDO

Godfrey, M., Hepburn, S., Fidler, D. J., Tapera, T., Zhang F., Rosenberg, C. R. y Raitano LEE, N. (2019). Autism spectrum disorder (ASD) symptom profiles of children with comorbid Down syndrome (Ds) and AsD: a comparison with children with Ds-only and AsD-only. Research in Developmental Disabilities, 89, 83-93. https://doi:10.1016/j.ridd.2019.03.003

Grieco, J., Pulsifer, M. B., Seligsohn, K., Skotko, B. G. y Schwartz, A. (2015). Down syndrome: cognitive and behavioral functioning across the lifespan. American Journal of Medical Genetics. Part C, Seminars in Medical Genetics, 169, 2, 135-149.

Hamner, T., Hepburn, S., Zhang, F., Fidler, D., Robbinson, C., Robins, D. y Lee, N. R. (2020). Cognitive profiles and autism symptoms in comorbid Down syndrome and Autism Spectrum Disorder. Journal of Developmental and Behavioral Pediatrics, 41(3), 172-179. https://doi:10.1097/Dвр.0000000000000745

Hartaway, S. A. (2016). El ajuste familiar al diagnóstico de autismo en el síndrome de Down. Revista Sindrome de Down, 33(131), 110-115.

Hepburn, S., Philofsky, A., Fidler, D. J. y Rogers, S. (2008). Autism symptoms in toddlers with Down syndrome: a descriptive study. Journal of Applied Research in Intellectual Disabilities, 21(1), 48-57. https://doi:10.1111/j.1468-3148.2007.00368.x

Hernández-Martínez, M., Pastor-Duran, X. y Navarro-Navarro, J. (2011). Trastorno por déficit de atención con o sin hiperactividad en los niños con síndrome de Down. Revista Internacional sobre el Sindrome de Down, 15(2), 18-22.

Howlin, P., Wing, L. y Gould, J. (1995). The recognition of autism in children with Down syndrome: implications for intervention and some speculations about pathology. Developmental Medicine and Child Neurology, 37(5), 406-414. https://doi:10.1111/j.1469-8749.1995. tb12024.x

Huete García, A. (2016). Demografía e inclusión social de las personas con síndrome de Down. Revista Sindrome de Down, 33, 38-50.

Ji, N. Y., Capone, G. T. y Kaufmann, W. E. (2011). Autism spectrum disorder in Down syndrome: cluster analysis of Aberrant Behaviour Checklist data supports diagnosis. Journal of Intellectual Disabilities Research, 55(11), 1064-1077. https://doi:10.1111/j.13652788.2011.01465.x

Karmiloff-Smith, A., Al-Janabi, T., D’Souza, H., Groet, J., Massand, E., Mok, K., Startin, C., Fisher, E., Hardy, J., Nizetic, D., Tybulewicz, V. y Strydom, A. (2016). The importance of understanding individual differences in Down syndrome. F1000research, 5. https://doi:10.12688/f1000research.7506.1

Kent, L., Evans, J., Paul, M. y Sharp, M. (1999). Comorbidity of Autistic Spectrum Disorders in children with Down syndrome. Developmental Medicine and Child Neurology, 41(3), 153-158. https://doi.org/10.1111/j.1469-8749.1999.tb00574.x

Korenromp, M. J., Page-Christiaens, G. C., van den Bout, J., Mulder, E. J. y Visser, G. H. (2007). Maternal decision to terminate pregnancy in case of Down syndrome. American Journal Obstetrics and Gynecology, 196(2), 149.e1-11. https://doi:10.1016/j. ajog.2006.09.013

Krieger, A. E., Lancéart, E., Nader-Grosbois, N. y Adrien, J. L. (2014). Trisomie 21 et autisme: double diagnostic, évaluation et intervention Down. Neuropsychiatrie de l'Enfance et de l'Adolescence, 62, 235-243.

Lovell, R. W. y ReIss, A. L. (1993). Dual diagnoses: psychiatric disorders in developmental disabilities. Pediatric Clinics of North America, 40(3), 579-592. https://doi.org/10.1016/ S0031-3955(16)38552-2 
APROXIMACIÓN AL ESTUDIO DE LAS DIFERENCIAS INDIVIDUALES Y AL DIAGNÓSTICO DE PATOLOGÍA DUAL DESDE LA PERSPECTIVA PSICOLÓGICA EN NIÑOS Y ADOLESCENTES CON SÍNDROME DE DOWN G. DELGADO-PARDO

Lund, J. (1988). Psychiatric aspects of Down's syndrome. Acta Psychiatrica Scandinavia, 78, 369-374.

MäÄttä, T., Tervo-MäÄttä, T., TaAnila, A., Kaski, M. e Iivanainen, M. (2006). Mental health, behaviour and intellectual abilities of people with Down syndrome. Down Syndrome Research and Practice, 11(1), 37-43.

Mantry, D., Cooper, S. A., Smiley, E., Morrison, J., Allan, L., Williamson, A., Finlayson, J. y Jackson, A. (2008). The prevalence and incidence of mental ill-health in adults with Down syndrome. Journal of Intellectual Disability Research, 52(Pt 2), 141-155. https:// doi:10.1111/j.1365-2788.2007.00985.x

Marino, M., Scala, I., Scicolone, O., Strisciuglio, P. y Bravaccio, C. (2019). Distribution and age of onset of psychopathological risk in a cohort of children with Down syndrome in developmental age. Italian Journal of Pediatrics, 45, 92. https://doi.org/10.1186/s13052019-0672-4

McCARTHY, J. (2008). Behaviour problems and adults with Down syndrome: childhood risk factors. Journal of Intellectual Disability Research, 52, Part. 10, 877-882. https://doi:10.1111/ j.1365-2788.2008.01104.x

Menolascino, F. J. (1965). Psychiatric aspects of mongolism. American Journal of Mental Deficiency, 69, 653-660.

Ministerio De Sanidad, Consumo Y Bienestar Social. (2017). Encuesta Nacional de Salud 2017. ENSE 2017. Disponible en: https://www.ine.es/metodología/tl5/tl53041917.pdf

Moss, J., Richards, C., Nelson, L. y Oliver, C. (2012). Prevalence of autism disorder symptomatology and related behavioural characteristics in individual with Down syndrome. Autism, 17(4), 390-404. https://doi:10.1177/1362361312442790

Myers, B. A. y Pueschel, S. M. (1991). Psychiatric disorders in persons with Down syndrome. The Journal of Nervous and Mental Disease, 179(10), 609-613.

Naerland, T., Bakke, K. A., Storvik, S., Warner, G. y Howlin, P. (2016). Age and genderrelated differences in emotional and behavioural problems and autistic features in children and adolescents with Down syndrome: a survey-based study of 674 individuals. Journal of Intellectual Disability Research, 61(6), 594-603. https://doi:10.1111/jir.12342

ORTiz, B. (2012). Los grupos terapéuticos de niños con patología dual: Síndrome de Down y rasgos autistas en la Fundació Catalana Síndrome de Down. Revista Médica Internacional sobre el Sindrome de Down, 16(3), 44-48. https://doi.org/10.1016/S1138-2074(12)70028-3

Ortiz, B., Videla, L., Gich, I., Alcacer, B., Torres, D., Jover, I., Sánchez, E., Iglesias, M., Fortea, J. y Videla, S. (2017). Señales de alarma precoces del trastorno del espectro autista en personas con síndrome de Down. Revista Médica Internacional sobre el Sindrome de Down, 21(1), 3-11. https://doi.org/10.1016/j.sd.2017.01.001

Oxelgren, U. W., Myrelid, Å., Annerén, G., Ekstam, B., Göransson, C., Holmbom, A., Isaksson, A., Åberg, M., Gustafsson, J. y Fernell, E. (2017). Prevalence of autism and attention-deficit-hyperactivity disorder in Down syndrome: A population-based study. Developmental Medicine and Child Neurology, 59(3), 276-283. https://doi:10.1111/ dmcn.13217. Epub 2016 Aug 9

Patterson, B. (1999). Dual diagnosis: the importance of diagnosis and treatment. Disability Solutions, 3, 16-17.

Pueschel, S. M. y Myers, B. A. (1994). Environmental and temperament assessments of children with Down's syndrome. Journal of Intellectual Disabilities Research, 38(Pt2), 195202. https://doi:10.1111/j.1365-2788.1994.tb00374.x

Ediciones Universidad de Salamanca / CC BY-NC-ND

Siglo Cero, vol. 52 (4), 2021, octubre-diciembre, pp. 131-154 
APROXIMACIÓN AL ESTUDIO DE LAS DIFERENCIAS INDIVIDUALES Y AL DIAGNÓSTICO DE PATOLOGÍA DUAL DESDE LA PERSPECTIVA PSICOLÓGICA EN NIÑOS Y ADOLESCENTES CON SÍNDROME DE DOWN G. DELGADO-PARDO

Reilly, C. (2009). Autism spectrum disorders in Down syndrome: a review. Research in $A u-$ tism Spectrum Disorders, 3, 829-839. https://doi.org/10.1016/j.rasd.2009.01.012

Rodgers, C. (1987). Maternal support for the Down's syndrome stereotype: the effect of direct experience of the condition. Journal of Mental Deficiency Research, 31, 271-278.

Rollin, H. R. (1946). Personality in mongolism with special reference to the incidence of catatonic psychosis. American Journal of Mental Deficiency, 51, 219-237.

Rondal, J. A., Hodapp, R., Soresi, S., Dykens, E. y Nota, L. (2004). Intellectual disabilities. Genetics, behavior and inclusion. X Preface. Whurr Publishers.

Rondal, J. A. y Ling, L. (2006). Especificidad neuroconductual en el síndrome de Down. Revista de Logopedia, Foniatría y Audiología, 26(1), 12-19.

Rosso, M., Fremion, E., Santoro, S. L., Oreskovic, N. M., Chitnis, T., Skotko, B. G. y SanTORO, J. D. (2020). Down Syndrome Disintegrative Disorder: a clinical regression syndrome of increasing importance. Pediatrics, 145(6), e20192939. https://doi:10.1542/peds.20192939

Rutter, M. y Schopler, E. (1988). Autism and pervasive developmental disorders: concepts and diagnostic issues. Journal of Autism and Developmental Disorders, 17, 159-186. https:// doi.org/10.1007/BF01495054

Santiago García, O. y Marrero Rodríguez, G. (2003). Actitudes hacia las personas con discapacidad en profesionales del Primer Ciclo de Educación Infantil. Anuario. Filosofía, Psicología y Sociología, 6, 131-158.

Santoro, S. L., Cannon, S., Capone, G., Franklin, C., Hart, S. J., Hobensack, V., Kishnani, P. S., Macklin, E. A., Manickam, K., Mccormick, A., Nash, P., Oreskovic, N. M., Patsiogiannis, V., Steingass, K., Torres, M., Valentini, D., Vellody, K. y Skotko, B. G. (2020). Unexplained regression in Down syndrome: 35 cases from an international Down syndrome database. Genetics in Medicine, 22(4), 767-776. https://doi:10.1038/s41436-0190706-8.

Spendelow, J. S. (2011). Assessment of mental health problems in people with Down syndrome: key considerations. British Journal of Learning Disabilities, 39, 306-313. https:// doi:10.1111/j.1468-3156.2010.00670.x

Startin, C. M., D’Souza, H., Ball, G., Hamburgo, S., Hithersay, R., Hughes, K. ... Strydom, A. (2020). Health comorbidities and cognitive abilities across the lifespan in Down syndrome. Journal of Neurodevelopmental Disorders, 12, 4. https://doi.org/10.1186/s11689-0199306-9

Stein, D. S., Munir, K. M., Karweck, A. J., Davidson, E. J. y Stein, M. T. (2013). Developmental regression, depression, and psychosocial stress in an adolescent with Down syndrome. Journal of Developmental and Behavioral Pediatrics, 34(3), 216-218. https://doi. org/10.1097/Dвр.0b013e31828b2b42

Troncoso, M. V. (2019). Sindrome de Down. Mi visión y presencia. Fundación Iberoamericana Down21 y CePe Editorial.

Troncoso, M. V., Del Cerro, M. y Ruiz, E. (1999). El desarrollo de las personas con SD: una visión longitudinal. Siglo Cero, 30(4), 184, 7-26.

Turk, J. (1992). Children with Down's syndrome and Fragile X syndrome: a comparison study. Society for the Study of Behavioural Phenotypes: 2 nd Symposium Abstracts.

Uppal, H., Chandran, S. y Potluri, R. (2015). Risk factors for mortality in Down syndrome. Journal of Intellectual Disability Research, 59(9), 873-881. https://doi:10.1111/jir.12196. Epub 2015 Apr 8. PMiD: 25851193 
Vicari, S., Pontillo, M. y Armando, M. (2013). Neurodevelopmental and psychiatric issues in Down's syndrome: assessment and intervention. Psychiatric Genetics, 23, Issue 3, 95 107. https://doi:10.1097/YPG.0b013e32835fe426

WakABAYASHI, S. (1979). A case of infantile autism associated with Down's syndrome. Journal of Autism and Developmental Disorders, 9, Issue 1, 31-36. https://doi.org/10.1007/ BF01531289

Walton, C. y Kerr, M. P. (2015). Down syndrome: systematic review of the prevalence and nature of presentation of unipolar depression. Advances in Mental Health and Intellectual Disabilities, 9(4), 151-162. https://dx.doi.org/10.1108/AмHid-11-20140037

Warner, G., Howlin, P., Salomone, E., Moss, J. y Charman, T. (2017). Profiles of children with Down syndrome who meet screening criteria for autism spectrum disorder (AsD): a comparison with children diagnosed with ASD attending specialist schools. Journal of Intellectual Disability Research, 61(1), 75-82. https://doi:10.1111/jir.12344.

Weijerman, M. E., van Furth, A. M., Vonk Noordegraaf, A., van Wouwe, J. P., Broers, C. J. y Gemke, R. J. (2008). Prevalence, neonatal characteristics, and first-year mortality of Down syndrome: a national study. The Journal of Pediatric, 152(1), 15-19. https:// doi:10.1016/j.jpeds.2007.09.045. Epub 2007 Nov 19. PMID: 18154890

WishaRt, J. G. y Johnston, F. H. (1990). The effects of experience on attribution of a stereotyped personality to children with Down's syndrome. Journal of Mental Deficiency Research, 34, 409-420. https://doi.org/10.1111/j.1365-2788 1990.tb01551.x

Worley, G., Crissman, B. G., Cadogan, E., Milleson, C., Adkins, D. W. y Kishnani, P. S. (2015). Down syndrome Disintegrative Disorder: new-onset autistic, dementia, and insomnia in older children and adolescents with Down syndrome. Journal of Child Neurology, 30(9), 1147-1152. https://doi:10.1177/0883073814554654

Yahia, S., El-Hadidy, M., El-Gilany, A. H., Amdel-Hady, D., Wahba, Y. y Al-Haggar, M. (2014). Disruptive behavior in Down syndrome children: a cross-sectional comparative study. Annals of Saudi Medicine, 34(6), 517-521. https://doi.org/10.5144/0256-4947.2014.517

Zickler, C. F., Morrow, J. D. y Bull, M. J. (1998). Infants with Down syndrome: a look at temperament. Journal of Pediatric Health Care, 12(3), 111-117. https://doi:10.1016/s08915245(98)90240-1 